\title{
Mottes, manoirs et châteaux : au-delà des inventaires archéologiques, l'exemple du Centre Ouest Bretagne
}

\author{
Patrick KERNEVEZ \\ Maître de conférences en histoire médiévale, Université de Bretagne occidentale, \\ Centre de Recherche Bretonne et Celtique (EA 4451)
}

Le thème des châteaux et des résidences aristocratiques du Moyen Âge a fait l'objet d'innombrables études depuis des décennies ${ }^{1}$. Michel de Boüard, " père de l'archéologie médiévale ", a même créé un néologisme au début des années 1960, celui de " castellologie ", forgé par la combinaison du terme latin castellum, château, et du grec logos, étude. Cette discipline combine les approches de plusieurs sciences : l'histoire par le biais des textes, l'archéologie par celui de la fouille et de la prospection, l'histoire de l'art par l'examen des caractéristiques architecturales. On n'étudie plus les châteaux par le seul biais de l'archéologie monumentale, de l'architecture militaire, de l'étude généalogique de ses propriétaires ou même des légendes et des traditions invérifiées. Le paradigme du château a été considérablement élargi : on attribue désormais à cet édifice une triple fonction, militaire, résidentielle et symbolique, ce qui a contribué à réorienter les travaux de recherche autour de thèmes, comme " demeurer, défendre et paraître ${ }^{2}$ ".

L'heure de la synthèse n'est pas encore venue pour le territoire breton où l'on recense environ 250 châteaux ${ }^{3}$, une soixantaine de villes " nées à l'ombre d'un château ${ }^{4}$ ", des centaines de mottes et d'enceintes de terre médiévales et des milliers de manoirs. Notre propos se limitera à l'examen des apports de cette historiographie récente ${ }^{5}$ dans le domaine de l'étude des mottes, des manoirs et des

${ }^{1}$ Des centaines de références sont citées par MESQUI, Jean, Châteaux et enceintes de la France médiévale, de la défense à la résidence, 2 vol., Paris, 1991 et 1993, t. II, p. 347-365.

${ }^{2}$ BouRgeols, Luc et RÉMY, Christian, (dir.), Demeurer, défendre et paraître. Orientations récentes de l'archéologie des fortifications et des résidences aristocratiques médiévales entre Loire et Pyrénées, actes du colloque de Chauvigny (14-16 juin 2012), Chauvigny, 2014.

${ }^{3}$ Une liste est donnée par АмІот, Christophe, Lignages et châteaux en Bretagne avant 1350, thèse de doctorat d'histoire, dactyl., 4 vol., Rennes, 1999, t. II, p. 688-693 et une carte réalisée par KERNÉVEZ, Patrick et AMIOT, Christophe, "Les châteaux bretons au Moyen Âge », dans TANGUY, Bernard et LAGRÉE, Michel, (dir.), Atlas d'histoire de Bretagne, Morlaix, 2002, p. 71.

${ }^{4}$ KeRnÉVEZ, Patrick et Le Gall-TANGuY, Régis, "Les châteaux et le peuplement en Basse-Bretagne : l'exemple des bourgs castraux de Cornouaille, Léon et Trégor ", dans CHÉDEVILle, André et PICHOt, Daniel, (dir.), Des villes à l'ombre des châteaux. Naissance et essor des villes castrales en France au Moyen Âge, actes du colloque de Vitré (16-17 octobre 2008), Rennes, 2010, p. 29-41.

${ }^{5}$ Consulter la synthèse récente de LAFFONT, Pierre Yves, "Le château médiéval en Bretagne. Un bilan historiographique ", dans LAFFONT, Pierre Yves, (dir.), Les élites et leurs résidences en Bretagne au Moyen Âge, actes du colloque de Guingamp - Dinan (28-29 mai 2010), Rennes, 2014, p. 113-123. 
châteaux appliquée à un territoire, celui du Centre Ouest Bretagne ${ }^{6}$ pour illustrer le chemin parcouru, les résultats obtenus et les défis à surmonter lors de l'étude conjointe de ces édifices qui correspondent parfois à une typologie que certains jugent désormais surannée.

\section{Le temps des inventaires : mottes et manoirs}

Hormis quelques précurseurs comme le chevalier de Fréminville, auteur de descriptions d'antiquités et de dessins concernant notamment des monuments disparus dans les années $1830-1840^{7}$, il faut attendre la fin du XIX siècle pour que soient réalisés des inventaires des sites archéologiques dans les différents départements bretons ${ }^{8}$. On peut notamment citer le travail de Henri Frotier de La Messelière auteur de plusieurs études sur les Côtes-d'Armor' ${ }^{9}$. Louis Le Guennec et le chanoine Abgrall sont les premiers à consacrer un article aux mottes du Finistère, en 1915, il y a tout juste un siècle, là où quelques années auparavant on ne voyait encore que la butte centrale de "camps romains ${ }^{10}$ ". Ces érudits nous ont laissé des notes quelquefois conservées dans des dépôts d'archives ${ }^{11}$ ainsi que des carnets de croquis et des dessins de nombreux monuments, petits ou grands, parfois disparus ${ }^{12}$. Les atlas féodaux de La Messelière témoignent ainsi de la domination des campagnes médiévales et modernes par une noblesse nombreuse, ce dont témoigne encore une profusion de manoirs, petits châteaux et retranchements de terre, témoins d'un véritable maillage seigneurial ${ }^{13}$.

${ }^{6}$ Le Pays du Centre Ouest Bretagne réunit 79 communes réparties sur cinq communautés de communes et trois départements, au centre de la Bretagne.

7 De FréminVILle, Christophe Paulin, Antiquités de Bretagne : Finistère, 2 vol., Brest, 1832 et 1835 ; ID., Guide du voyageur dans le Finistère, Brest, 1844. Citons notamment les châteaux du Breignou en Bourg-Blanc, Kermilin en Tréflaouénan et Quimerc'h en Bannalec.

${ }^{8}$ Pour le Finistère: LE MEN, René François, « Statistique monumentale du Finistère, époque romaine », Bulletin de la Société archéologique du Finistère, t. II, 1874-1875, p. 122147 ; ID., "Statistique monumentale du Finistère (époque celtique) », Ibid., t. III, 1876, p. 85136 ; FlAGELLE, Édouard, « Notes archéologiques sur le département du Finistère », Bulletin de la Société académique de Brest, $2^{\text {ème }}$ série, t. IV, 1876-1877, p. 1-90 ; Du CHÂTELLIER, Paul, Les époques préhistoriques et gauloises dans le département du Finistère, $2^{\text {ème }}$ éd., Rennes-Quimper, 1907.

${ }^{9}$ FROTIER DE LA MESSELIĖRE, Henri, « De l'âge probable des châteaux de terre des Côtesdu-Nord », Bulletin de la Société d'émulation des Côtes-du-Nord, t. LXV, 1933, p. 51-79.

10 Abgrall, Jean-Marie, "Mottes féodales », Bulletin de la Société archéologique du Finistère, t. XLII, 1915, p. 54-85 ; LE GUENNEC, Louis, "Les mottes féodales du pays de Morlaix », Ibid., p. 86-105.

11 Sous-séries $60 \mathrm{~J}$ des Archives départementales des Côtes-d'Armor (H. Frotier de la Messelière) et $34 \mathrm{~J}$ des Archives départementales du Finistère (L. Le Guennec).

${ }^{12}$ Un aperçu dans l'article de DROGUET, Alain, « Les archives départementales des Côtesd'Armor », dans Mignot, Claude et CHATENET, Monique, (dir.), Le manoir en Bretagne 13801600, Paris, 1993, p. 288-295 (p. 294-295).

${ }^{13}$ Voir pour exemple l'atlas féodal d'Henri Frotier de la Messelière comprenant 19 cartes au $1 / 50000^{\mathrm{e}}$ tracées à la plume aux Archives départementales des Côtes-d'Armor, soussérie $60 \mathrm{~J}$. 
Ces inventaires archéologiques, quoiqu'incomplets et imprécis, constituent toujours le fondement de l'actuelle carte archéologique. Ils ont été prolongés par des études universitaires comme celle consacrée dès 1967 aux "mottes féodales " du Finistère et d'autres travaux initiés dans les années 1980 sur les mottes d'llle-etVilaine, les châteaux de haute Bretagne et les fortifications médiévales du Léon ${ }^{14}$. Dans les années 1990, sous l'égide de la Section archéologie de l'Institut Culturel de Bretagne ont été réalisés des inventaires des mottes d'llle-et-Vilaine en 1990, des mottes des Côtes-d'Armor en 1994, des fortifications du haut Moyen Âge en Bretagne, ainsi que des fortifications médiévales du Finistère en $1997^{15}$. On a ainsi privilégié l'étude d'un type d'ouvrage, la motte, au point de laisser croire qu'il était l'archétype du château des $\mathrm{XI}^{\mathrm{e}}$ et XII ${ }^{\mathrm{e}}$ siècles, voire, vers l'an mil, « une arme pour une révolution ${ }^{16} »$. Seul l'inventaire consacré au Finistère a pris en compte les autres types d'ouvrages: enceintes de terre circulaires, ouvrages protohistoriques réoccupés, maisons fortes et châteaux de pierre. Ces recensions sont restées incomplètes, notamment dans le Morbihan, en dépit de deux travaux universitaires menés en 1993 et $2002^{17}$, ceci jusqu'au doctorat d'archéologie de Lucie Jeanneret, soutenu en $2016^{18}$. Des lacunes subsistent pour certains secteurs éloignés des villes à partir desquelles opéraient les anciens prospecteurs. Les bois et les forêts n'ont pas été systématiquement explorés pas plus que n'ont été exhaustivement dépouillés les atlas et les états des sections des cadastres napoléoniens pour y traquer des parcellaires révélateurs et des indices micro-toponymiques ${ }^{19}$. Des découvertes fortuites ou des prospections menées sur des secteurs à l'échelle

${ }^{14}$ LE HAN, Roger, Les mottes féodales dans le Finistère, D.E.S. d'histoire médiévale, dactyl., Brest, 1967 ; Амıот, Chistophe, Châteaux de la Bretagne médiévale : comté de Rennes, partie nord, et comté de Penthièvre, 5 vol., mémoire de maîtrise d'histoire de l'art, dactyl., Rennes, 1986 ; BRAND'HONNEUR, Michel, Les mottes dans la baronnie de Vitré à la lumière de l'archéologie et des textes, mémoire de maîtrise d'histoire, dactyl., 2 vol., Rennes, 1988 ; KERNÉVEZ, Patrick, Châteaux et fortifications du Comté de Léon (XI siècle - milieu du XIV siècle), mémoire de maîtrise d'histoire, dactyl., 2 vol., Brest, 1988.

15 BRAND'HonneUR, Michel, Les mottes médiévales d'Ille-et-Vilaine, Rennes, 1990 ; HINGANT, Stephan, Les mottes médiévales des Côtes-d'Armor, Rennes, 1994 ; GuIGON, Philippe, Les fortifications du haut Moyen Âge en Bretagne, Rennes, 1997 ; KERNÉVEZ, Patrick, Les fortifications médiévales du Finistère, mottes, enceintes et châteaux, Rennes, 1997.

${ }^{16}$ BUR, Michel, "Vers l'an Mil, la motte, une arme pour une révolution », L'information historique, t. 44/3, 1982, p. 101-108.

17 Pesnel, Laurent, Les mottes castrales et les enceintes circulaires du Morbihan occidental. $X{ }^{e}-X I I^{e}$ siècle, mémoire de maîtrise d'histoire, dactyl., Brest, 1993 ; LE BADEZET, Nicolas, Mottes et enceintes du département du Morbihan (XI ${ }^{e}$-XIII ${ }^{e}$ siècles), mémoire de DEA d'histoire, 2 vol., dactyl, Rennes, 2002.

18 JeANNERET, Lucie, L'habitat fortifié et fossoyé dans le Vannetais et le Porhoët : étude de la structuration des pouvoirs et du peuplement au Moyen Âge ( $X^{e}-X I I I^{e}$ siècle), doctorat d'archéologie, dactyl., Rennes, 2016.

${ }^{19}$ Sur la méthodologie adoptée, voir KERNÉVEZ, Patrick, Les fortifications médiévales du Finistère..., op. cit., p. 11-14. 
cantonale $\mathrm{e}^{20}$ ou même communale $\mathrm{e}^{21}$ ont parfois permis de découvrir des ouvrages ou des indices de sites inédits. L'archéologie aérienne apporte des données complémentaires, même si les résultats concernant la période médiévale sont a priori moins notables que pour des périodes antérieures ${ }^{22}$. La datation d'une partie des sites repérés reste indéterminée avec, notamment, des hésitations entre l'Âge du Fer et le Moyen Âge que seule une démarche comparative et une prospection au sol peuvent partiellement lever ${ }^{23}$.

Ces travaux focalisés sur un "brun manteau de mottes ${ }^{24}$ " ont abouti à une vision tronquée du phénomène castral. On a ainsi pu cartographier d'une part les mottes et d'autre part les châteaux sans nécessairement les mettre en relation. Pire encore, certains châteaux urbains totalement disparus n'ont pas été pris en compte alors qu'ils avaient généré, ou avaient contribué à générer, le développement d'agglomérations castrales. En outre, on a peu cherché à mettre en parallèle l'étude des mottes avec celle des manoirs ${ }^{25}$ : la première relève du travail des archéologues et des prospecteurs alors que la seconde est bien souvent menée par des historiens, des architectes et l'Inventaire général qui a pu réaliser certains travaux exhaustifs dès lors que les monuments étaient bien conservés et documentés. II en fut ainsi pour les inventaires topographiques des cantons de Carhaix-Plouguer, Le Faouët et Gourin, publiés en 1969 et $1975^{26}$ avant qu'une synthèse ne soit consacrée au manoir breton en $1993^{27}$. De nombreux édifices ont par ailleurs bénéficié des approches conjointes de Gwyn Meirion-Jones et de Michael Jones, associant examen du bâti et analyse des textes ${ }^{28}$. Parallèlement, Jean Kerhervé a fait travailler

${ }^{20}$ LE QUELLEC, Vincent, Système d'informations géographiques appliqué à un inventaire critique et analytique des sites fossoyés et/ou fortifiés en terre du Porzay (Finistère), mémoire de maîtrise d'archéologie, dactyl., 3 vol., Paris, 2004 ; ID., LE GofFIC, Michel, PEUZIAT, Josick, "Les enceintes médiévales du Porzay (Finistère) », Bulletin de la Société archéologique du Finistère, t. CXXXV, 2006, p. 37-69.

21 TAnguY, Daniel, Arzano. Archéologie du paysage, mémoire de maîtrise d'histoire, dactyl., Brest, 1982.

${ }^{22}$ GAUTIER, Maurice, «Des centaines de sites vus d'avion », dans MÉNEZ, Yves, LORHO, Thierry et ChARTIER-Le FLOCH, Erwan, (dir.), Archéologie en centre Bretagne, Spézet, 2015, p. 18-19.

${ }^{23}$ II en était ainsi pour le Camp des Salles à Locronan que Pierre Rolland Giot attribua au Moyen Âge, datation confirmée lors des fouilles de Philippe Guigon (GUIGON, Philippe, Les fortifications du haut Moyen Âge..., op. cit., p. 69-98) Consulter LE GALL, Joseph et LEROY, Benjamin, "Le haut Moyen Âge », dans Archéologie en Centre Bretagne..., op. cit., p. 126140 (p. 129-133) et l'article de Nicolas LE BADEZET dans le présent ouvrage.

${ }^{24}$ Expression forgée par MORSEL, Joseph, L'aristocratie médiévale $V^{e}-X V^{e}$ siècle, Paris, 2004, p. 90.

${ }^{25}$ À l'exception notable de Brand'HONneUR, Michel, Manoirs et châteaux dans le comté de Rennes. Habitat à motte et société chevaleresque (XI $I^{e}$, XII siècles), Rennes, 2001.

${ }^{26}$ Inventaire général des monuments et des richesses artistiques de la France. Région Bretagne, Finistère, Canton Carhaix-Plouguer, Paris, 1969 ; ID., Morbihan, Cantons Le Faouët et Gourin, Paris, 1975.

${ }^{27}$ Mignot, Claude et Chatenet, Monique, (dir.), Le manoir en Bretagne..., op. cit.

${ }^{28}$ Au sein d'une abondante bibliographie, citons MEIRION-JONES, Gwyn, JONES, Michael et GuIBAL, Frédéric, "La résidence noble en Bretagne, $\mathrm{XI}^{\mathrm{e}}-\mathrm{XVI} \mathrm{l}^{\mathrm{e}}$ siècle. Salles, chambres et tours ", dans MEIRION-JONES, Gwyn, (dir.), La demeure seigneuriale dans l'espace Plantagenêt, salles, chambres et tours, Rennes, 2013. Des mêmes auteurs, consulter de 
des étudiants de l'université de Bretagne occidentale sur des inventaires de manoirs menés à l'échelle d'un canton : le travail initié par la transcription des réformations de la noblesse des $X V^{e}$ et $X V I^{e}$ siècles a été poursuivi par des investigations en archives et sur le terrain pour rendre compte du paysage manorial passé et présent ${ }^{29}$. La cartographie des études menées parallèlement à la poursuite des travaux de l'Inventaire témoigne de l'importance du phénomène manorial en Bretagne et tout autant de l'ampleur des destructions opérées depuis le $\mathrm{XVl}^{\mathrm{e}}$ siècle. Certains manoirs ont fait l'objet de monographies, d'articles dans des revues spécialisées ${ }^{30}$ ou même d'ouvrages, comme ceux d'Yves Lulzac ${ }^{31}$ ou de Jean-Yves Le Goff ${ }^{32}$ pour les édifices léonards. II convient d'en tenir compte, en dépit du caractère souvent généalogique de certaines études. On doit également saluer le travail d'Hervé Torchet qui a publié des registres de réformation et des montres de la noblesse du $\mathrm{XV}^{\mathrm{e}}$ siècle et contribué au réexamen de certaines généalogies ${ }^{33}$.

belles monographies de manoirs dans les Mémoires de la Société d'histoire et d'archéologie de Bretagne, avec des mises en perspective du travail en archives et d'examen du bâti.

${ }^{29}$ CRÉAC'H, Matthieu, Les manoirs du Léon occidental entre l'Aber-Benoît et l'Aber-lldut, mémoire de maîtrise d'histoire, dactyl., Brest, 1991 ; CLÉAC'H, Thierry, Les sources de I'histoire des manoirs aux $X V^{e}$ et $X V l^{e}$ siècles : l'exemple du pays de Lesneven, mémoire de maîtrise d'histoire, dactyl., Brest, 1991 ; LouBoutIN, Ronan, Les manoirs de deux pays de Kerne entre 1400 et 1600, la basse vallée de l'Aulne et le Porzay, mémoire de maîtrise d'histoire, dactyl., Brest, 1993 ; PICHOURON, Patrick, Manoirs et propriétaires aux XV et $X V l^{e}$ siècles dans le régaire de Tréguier et la seigneurie de Botloy-Lezardre, mémoire de maîtrise d'histoire, dactyl., Brest, 1994 ; GuILLOU, Jean-Philippe, Manoir et société dans la sénéchaussée de Brest et Saint-Renan (paroisses de Plouzané, Plougonvelin, Ploumoguer, Plouarzel, Saint-Renan), mémoire de maîtrise d'histoire, dactyl., Brest, 1994 ; PARC, Isabelle, Les manoirs des pays des Abers auX XV $\mathrm{V}^{e}$ et $X V l^{e}$ siècles (communes de Guissény, Landéda, Lannilis, Plouguerneau, Saint-Frégant), mémoire de maîtrise d'histoire, dactyl., Brest, 1996 ; LE GALL, Myriam, Les manoirs de la presqu'île saint-politaine auX $X V^{e}$ et $X V l^{e}$ siècles (communes de l'île de Batz, Plouénan, Plougoulm, Roscoff, Saint-Pol-de-Léon, Santec, Sibiril), mémoire de maîtrise d'histoire, dactyl., Brest, 1997 ; NouRRY, Audrey, Les manoirs des $X V^{\ominus}$ et $X V l^{e}$ siècles au cœur du Léon (communes de Bodilis, Cléder, Lanhouarneau, Mespaul, Plouescat, Plougar, Plounévez-Lochrist, Plouvorn, Plouzévédé, Saint-Vougay, Tréflaouénan, Tréflez, Trézilidé), mémoire de maîtrise d'histoire, dactyl., Brest, 1997 ; SCOUARNEC, Morgane, Les manoirs des $X V^{e}$ et $X V l^{e}$ siècles du canton de Plouigneau, mémoire de maîtrise d'histoire, dactyl., Brest, 1998 ; LE BOENNEC, Nicolas, Les manoirs des $X V^{\ominus}$ et $X V l^{e}$ siècles dans le sud du pays bigouden, mémoire de maîtrise d'histoire, dactyl., Brest, 2004.

${ }^{30}$ Comme la revue Manoirs et vieilles demeures en Bretagne.

${ }^{31}$ LULZAC, Yves, Chroniques oubliées des manoirs bretons, contribution à l'histoire des manoirs nobles du Bas-Léon sous l'Ancien Régime, 5 vol., Nantes, 1994-2005.

${ }^{32}$ LE GofF, Jean-Yves, Châteaux et manoirs du canton de Saint-Pol-de-Léon, Quimper, 1989 ; ID., Châteaux et manoirs du canton de Landivisiau, Quimper, 2003 ; ID., Châteaux et manoirs du canton de Plouzévédé, Quimper, 2006 ; ID., Châteaux et manoirs du canton de Taulé, Quimper, 2007.

${ }^{33}$ TORCHET, Hervé et TORCHET, Yann, Réformation des fouages de 1426. Diocèse ou évêché de Cornouaille, 2 vol., Paris, 2002 ; TORCHET, Hervé, Réformation des fouages de 1426 : diocèse ou évêché de Tréguier, 2 vol., Paris, 2003 ; ID., Réformation des fouages de 1426, évêché de Saint-Malo, Paris, 2005 ; ID., Réformation des fouages de 1426 : diocèse ou évêché de Léon, 2 vol., Paris, 2010. 


\section{Les questionnements des castellologues}

L'archéologie médiévale a connu d'importants développements depuis sa naissance et son affirmation en France, sous l'égide du doyen Michel de Boüard, dans les années 1950-1960. De nombreux champs de recherche sont alors apparus, notamment pour les châteaux. Une rapide évocation historiographique permet de mesurer les progrès accomplis et les interrogations qui en résultent ${ }^{34}$. La castellologie française est née à l'occasion de quelques chantiers qui ont fait date (Caen, Calvados ; Doué-la-Fontaine, Maine-et-Loire; Andone, Charente...), de l'impulsion donnée par le Centre de Recherches Archéologiques Médiévales ${ }^{35}$ et quelques autres équipes ainsi que des colloques internationaux bisannuels de Château Gaillard, à partir de $1962^{36}$. En 1980, s'est tenu à Caen un colloque consacré aux fortifications de terre en Europe occidentale, avec trois thèmes : la motte, les enceintes circulaires médiévales, châteaux et pouvoirs de commandement ${ }^{37}$. On a alors initié un ambitieux programme de recension nationale des milliers de fortifications de terre qui a fait long feu ${ }^{38}$. II en a toutefois résulté de nombreux inventaires et des thèses associant travail sur les sources et prospections de terrain sur des thématiques "Châteaux, pouvoirs et peuplement ". Deux programmes du conseil national de la recherche archéologique (CNRA) furent alors consacrés aux châteaux, H 39 pour les «châteaux forts » et H 40 pour les "fortifications de terre " avant d'être réunis dans un unique programme $\mathrm{H} 17$ " naissance, évolution et fonctions du château médiéval », en 1990. Des discussions ont porté sur la typologie des ouvrages puis sur la définition de ce qu'était une maison forte, catégorie d'ouvrage se situant entre les mottes, les simples maisons nobles et les " véritables châteaux $\mathrm{F}^{39}$ " puis sur les bourgs castraux, agglomérations nées à proximité des châteaux ${ }^{40}$. Faute de réel consensus, du fait de l'ampleur de la tâche et en raison de la disparition prématurée de certains acteurs (A. Debord, J.-M.

34 BouChARLAT, Élise, "Panorama de l'archéologie castrale en France », Revue archéologique du centre de la France, t. 48, 2009, consulté en ligne le 12 juillet 2013, http://racf.revues.org/1353; LEROUX, Laure, «Du monument à son histoire : aperçu méthodologique des études castrales en France », Annales de Janua, n 1, 2013, consulté en ligne le 15 avril 2013, http://annalesdejanua.edel.univ-poitiers.fr/index.php?id=184.

${ }^{35}$ Devenu le CRAHAM, Centre de recherches archéologiques et historiques anciennes et médiévales ou Centre Michel de Boüard (UMR 6273).

${ }^{36}$ Par exemple, les actes du colloque de Houffalize (Belgique) en septembre 2006, consacré au Bilan des recherches en castellologie: Château Gaillard: études de castellologie médiévale, t. XXIII, Caen, 2008.

37 BOÜARD, Michel de, "La motte »; DECAËNS, Joseph, "Les enceintes circulaires médiévales "; DEBORD, André, "Château et pouvoirs de commandement ", dans Les fortifications de terre en Europe occidentale $d u X^{e}$ au XII siècle, actes du colloque de Caen (2-5 octobre 1980), Archéologie Médiévale, t. XI, 1981, p. 5-123.

38 PESEZ, Jean-Marie, "Approche méthodologique d'un recensement général des fortifications de terre médiévales en France », Château Gaillard, t. XII, 1985, p. 79-90.

39 BuR, Michel, (dir.), La maison forte au Moyen Âge, actes de la table ronde de Nancy/Pont-à-Mousson (31 mai - 3 juin 1984), Paris, 1986.

${ }^{40}$ ID. (dir.), Les peuplements castraux dans les pays de l'Entre-Deux : Alsace, Bourgogne, Champagne, Franche-Comté, Lorraine, Luxembourg, Rhénanie-Palatinat, Sarre, actes du colloque de Nancy (1-3 octobre 1992), Nancy, 1993. 
Pesez) le travail sur les fortifications de terre et sur les bourgs castraux n'a pas été mené à son terme et les recherches sur les mottes se sont essoufflées.

Dans le même temps, l'archéologie a connu de profondes mutations avec la professionnalisation de la profession et la loi de 2001 sur l'archéologie préventive. On a pu déplorer la diminution du nombre des opérations menées sur les châteaux, notamment des fouilles programmées, et le fait qu'elles soient plus ponctuelles, liées à des travaux d'aménagement circonscrits dans l'espace et la durée, davantage réalisées en milieu urbain ou à l'occasion de travaux sur des monuments historiques, et qu'elles ne portent plus guère sur les origines des châteaux ${ }^{41}$. Le tableau peut être nuancé grâce aux enseignements de certaines fouilles conduites sur la longue durée (Charavines, Isère ; Boves, Somme ; Château-Thierry, Aisne...), la publication de travaux exemplaires (Andone, Charente), le choix fait par certaines collectivités de valoriser leur patrimoine castral, de pair avec des opérations archéologiques d'envergure (Falaise, Calvados ; Chinon, Indre-et-Loire ; Mayenne, Mayenne...) ou encore l'apport indéniable de certaines fouilles préventives entreprises avec des moyens importants (Pinheuil, Gironde). Des Programmes Collectifs de Recherche (PCR), ont été par ailleurs initiés, comme celui sur les enceintes médiévales dans le grand Ouest ${ }^{42}$.

Certains universitaires considèrent néanmoins aujourd'hui que, en quête de légitimité, les archéologues ont été engagés, malgré eux, dans le débat historiographique opposant les historiens des sources écrites sur la « mutation féodale » ou "révolution de l'an mil » avec la multiplication des inventaires de mottes dont le nombre ne pouvait qu'accréditer les thèses relatives à l'" anarchie féodale ${ }^{43}$. Florence Journot a ainsi dénoncé la "subordination aux problématiques historiques ", déploré que le château soit désormais un "concept fourre-tout » et jugé que les tentatives morpho-typologiques ou « typomanie » dissociant les mottes, les enceintes circulaires et les plates-formes de maisons fortes étaient non opératoires $^{44}$. On emprunte maintenant parfois au vocabulaire de la sociologie et de l'ethnologie en évoquant des " habitats aristocratiques ", " habitats seigneuriaux" ou encore "habitats des élites ". Dans une récente synthèse, Annie Renoux préfère évoquer des "Châteaux, palais et habitats aristocratiques fortifiés et semifortifiés ${ }^{45}$ ". Après avoir fait de la motte "la pièce emblématique de la révolution de l'an $\mathrm{Mil}^{46}$ » et parfois la résidence des chevaliers, on en vient aujourd'hui à considérer

${ }^{41}$ RenOuX, Annie, "Châteaux, palais et habitats aristocratiques fortifiés et semi-fortifiés ", dans ChAPELOT, Jean, (dir.), Trente ans d'archéologie médiévale en France. Un bilan pour un avenir, actes du congrès de Vincennes (16-18 juin 2006), Caen, 2010, p. 239-256 ; ID., "Châteaux, palais et habitats aristocratiques fortifiés ", Dossiers d'archéologie, $n^{\circ} 314$, juin 2006, p. $72-77$.

${ }^{42}$ Martineau, Jocelyn, (dir.), Projet collectif de recherche. Enceintes médiévales dans le Grand Ouest, dactyl., Nantes, 2011.

${ }^{43}$ Sur le paradigme historiographique de la « mutation de l'an mil », voir la synthèse de MAZEL, Florian, Féodalités 888-1180, Paris, 2010, p. 637-648.

44 JOURNOT, Florence, "Que sont châteaux et églises d'antan devenus ? ", dans Burnouf, Joëlle, ARribet-Deroin, Danielle, Desachy, Bruno, Journot, Florence, NissenJAUBERT, Anne, Manuel d'archéologie médiévale et moderne, Paris, 2009, p. 275-281 ; ID., "Le "château" à l'épreuve de la programmation archéologique ", Archéopages, n 19, août 2007, p. 50-53 (p. 50).

${ }^{45}$ Renoux, Annie, « Châteaux, palais et habitats aristocratiques... », art. cité, p. 239.

${ }^{46}$ JOURNOT, Florence, « Que sont châteaux et églises d'antan... ? », art. cité, p. 280. 
que l'on a à la fois surinterprété les textes et les données de prospection sans fouiller des sites, en associant leur première mention avec des ouvrages observables, peutêtre postérieurs. On en conclut désormais que la norme pour les élites rurales serait, durant tout le Moyen Âge, celle d'un «habitat à plat», plus commode. Le perchement, sous forme de motte, de tour correspondant à une résidence verticalisée ou de roca dans les reliefs du Midi, serait une exception manifestant la volonté de voir et d'être vu, de surveillance et d'ostentation, particulièrement aux $\mathrm{XI}^{\mathrm{e}}$ et $\mathrm{XII}^{\mathrm{e}}$ siècles. Hervé Mouillebouche, auteur d'un remarquable inventaire des châteaux bourguignons ${ }^{47}$, peut ainsi affirmer que les vraies mottes sont rares et l'ont toujours été, et considérer que : "Les inventaires menés en Bretagne, en Normandie ou en Bourbonnais, et qui ont pu accréditer l'idée d'un vaste mouvement d'emmottement, sont souvent des inventaires de chimères ${ }^{48} »$. On peut bien sûr s'offusquer du propos mais on se doit d'en tenir compte et reporter le chiffre de la centaine de mottes certaines recensées dans le Finistère aux quelque 3500 manoirs que l'on a pu y inventorier ${ }^{49}$. Même en multipliant le nombre de mottes par trois, en tenant compte des sites probables, hypothétiques et totalement détruits, on ne dépassera guère $10 \%$ du total envisagé des résidences aristocratiques, entre le $X^{\mathrm{e}}$ et le $\mathrm{XVI} l^{\mathrm{e}}$ siècle.

De fait, on a renoncé à voir dans la motte l'ancêtre du « château-fort » et seule une trentaine de celles-ci pourraient réellement avoir joué un rôle militaire ou politique à l'échelle du Finistère et être qualifiées de " castrales $^{50}$ ». Le plus grand nombre doit être considéré comme une manifestation ostentatoire de pouvoir qui a pu résulter de l'imitation des tertres les plus importants détenus par des seigneurs châtelains. Elles ont pu être érigées jusqu'au XIII ${ }^{\mathrm{e}}$ siècle, avec leur accord, aussi bien qu'à l'occasion d'un affaiblissement ponctuel de leur autorité lors du "choc châtelain », vers le $\mathrm{Xl}^{\mathrm{e}}$ siècle. Dans le comté de Rennes, Michel Brand'honneur ${ }^{51}$ évoque des «manoirs à motte " pour illustrer ces constructions avant tout ostentatoires et, dans le Maine, Annie Renoux suggère qu'il y a eu plusieurs générations de mottes, avec notamment des tertres érigés très tardivement à proximité d' " hébergements " comme témoignage de la pseudo " antiquité " de certains fiefs et volonté de se positionner hiérarchiquement ${ }^{52}$. Ces ouvrages qu'elle

47 Mouillebouche, Hervé, Les maisons fortes en Bourgogne du Nord du XIII au XVe siècle, Dijon, 2002.

${ }^{48}$ ID., « "L'inventaire des châteaux bourguignons: bilan et perspectives », Bulletin du centre d'études médiévales d'Auxerre, 2007, consulté en ligne le 13 février 2014, http://cem.revues.org/1097 ; DOI : 10.4000/cem.1097, p. 8.

49 DouARD, Christel, "Phénomènes de densité à moyenne et grande échelle ", dans Mignot, Claude et ChATEnet, Monique, (dir.), Le manoir en Bretagne..., op. cit., p. 45-53 (p. 53).

50 KeRnÉVEZ, Patrick, "Mottes et châteaux du département du Finistère », dans QuAghebeUR, Joëlle et SoleIL, Sylvain, (dir.), Le pouvoir et la foi au Moyen Âge en Bretagne et dans l'Europe de l'ouest. Mélanges en mémoire du professeur Hubert Guillotel, Rennes, 2010, p. 459-474.

${ }^{51}$ BRAnd'HOnNeUR, Michel, Manoirs et châteaux dans le comté de Rennes..., op. cit.

${ }^{52}$ RENOUX, Annie, « De la « motte » à l'hébergement fossoyé : étude de cas manceaux $\left(X l^{e}-X V l^{e}\right.$ siècle) », dans LALOU, Élisabeth, LEPEUPLE, Bruno et RoCH, Jean-Louis, (dir.), Des châteaux et des sources. Archéologie et histoire dans la Normandie médiévale : mélanges en l'honneur d'Anne-Marie Flambard-Héricher, Rouen, 2008, p. 161-185. 
qualifie parfois de "mottes plates " seraient avant tout des marqueurs sociaux, au même titre que des éléments défensifs ajoutés à certaines demeures nobles.

Bon nombre de mottes, mais aussi d'enceintes circulaires de terre, ont pu précéder un manoir dont elles ne sont distantes que de quelques dizaines ou centaines de mètres, tout comme du moulin qui porte le même nom. Le cas des enceintes de terre circulaires est parfois significatif : elles ont été érigées sur une période bien plus longue que les mottes comme l'ont démontré quelques fouilles récentes en Bretagne. Certaines, parfois érigées dès l'époque carolingienne, sont indéniablement à l'origine de manoirs. La fouille de Bressilien à Paule (Côtes d'Armor), non loin de l'important site gaulois de Saint-Symphorien, a révélé que cet enclos abritait une résidence aristocratique et ses dépendances des $\mathrm{VII}^{\mathrm{e}}, \mathrm{VIII}^{\mathrm{e}}$ et IX $X^{\mathrm{e}}$ siècles. À proximité, on a découvert les vestiges d'un manoir d'une trentaine de mètres de longueur, détruit au XIV siècle, qui lui a sans doute succédé ${ }^{53}$. Le site de La Montagne à Visseiche (Ille-et-Vilaine) a été découvert par l'archéologie aérienne : sa fouille a montré que des enclos qui passaient pour protohistoriques correspondaient en fait à un "manoir à enceintes fossoyées, accolées et plates » des $X I V^{e}$ et $X V^{e}$ siècles, ancêtre d'une autre résidence des $X V^{e}$ et $X V I^{e}$ siècles édifiée à 300 mètres de là ${ }^{54}$. Un paysage manorial fut aménagé au $X V^{e}$ siècle, faisant disparaître toute trace de cet ouvrage implanté dans une position éminente où l'on a par ailleurs détecté la présence d'un enclos d'habitat gaulois et de tombes de l'Âge du Bronze. Ces deux exemples laissent envisager une part de ce qui reste à découvrir en matière de résidence aristocratique de la moyenne et la petite noblesse rurale, par le biais de l'archéologie aérienne et de la fouille méthodique, hors des archétypes de la motte de terre et de bois des $\mathrm{XI}^{\mathrm{e}}$ et $\mathrm{XII}^{\mathrm{e}}$ siècles, et du manoir de pierre des $\mathrm{XV}^{\mathrm{e}}$ et $\mathrm{XVI} \mathrm{l}^{\mathrm{e}}$ siècles. Ils suggèrent également de possibles pérennités d'occupation de terroirs fertiles par des habitats de type aristocratique durant des millénaires. Jean-Claude Meuret établit ainsi des parallèles entre les manoirs à motte et les enclos du second Âge du Fer qui peuvent présenter des plans similaires avec une structure bipartite associant cour, basse-cour et mise en scène de l'entrée sous forme de tour-porte. Yves Ménez, qui a fouillé l'habitat aristocratique gaulois et l'oppidum de Paule, emprunte le vocabulaire des médiévistes en évoquant successivement une " maison forte ", un "château fort " et un "bourg castral " pour décrire le passage d'une demeure aristocratique rurale à une résidence fortifiée puis à une agglomération associée ${ }^{55}$.

Pour ce qui concerne les inventaires, il est certain que le qualitatif doit rivaliser avec le quantitatif. Le nombre de "sites aristocratiques" recensés dans un département peut atteindre plusieurs centaines. Pour mémoire, en 1997, dans le Finistère nous avons inventorié 438 "fortifications médiévales » : 43 mottes à bassecour, 48 mottes certaines, 28 mottes probables, 63 mottes hypothétiques, 83 enceintes circulaires, 37 enceintes quadrangulaires, 22 enceintes réoccupées au Moyen Âge, 39 enceintes détruites, 9 enceintes associées à des manoirs, 42

${ }^{53}$ MeURET, Jean-Claude, « Origines et débuts du manoir. Quelques observations pour la Bretagne, le Maine et l'Anjou ", dans La demeure seigneuriale dans l'espace Plantagenêt..., op. cit., p. 67-94 (p. 77), d'après LE GALL, Joseph et MÉNEZ, Yves, L'enceinte de Bressilien à Paule (Côtes-d'Armor). Rapport intermédiaire de fouille bisanuelle, 2009-2010, Conseil général des Côtes-d'Armor, Service régional de l'archéologie de Bretagne, 2010.

${ }^{54}$ ID., ibid., p. 84-88.

${ }^{55}$ MÉNEZ, Yves, "L'enceinte de Paule », dans Archéologie en centre Bretagne, op. cit., p. $90-92$ (p. 92). 
châteaux de pierre, 19 châteaux mal connus, 11 enceintes de pierre et 26 maisons fortes ou tours. Cet inventaire à la Prévert peut bien entendu donner le tournis et correspondre à ce qu'Annie Renoux désigne comme un « semis castral dense, mais surévalué ${ }^{56}$ ". II convient de le comparer à ceux d'autres régions pour démontrer qu'il est encore incomplet mais peut-être pas si critiquable: Charles Laurent Salch a recensé anciennement jusqu'à 30000 châteaux et sites fortifiés médiévaux en France $^{57}$. Des inventaires récents font état de plus de 200 donjons, tours et châteaux dans le département du Lot, 450 châteaux « romans » en Poitou-Charentes et 2600 châteaux en Bourgogne ${ }^{58}$. On ne doit cesser en outre de rappeler que les chronologies d'occupation diffèrent, certains sites n'ayant connu qu'une présence temporaire, et toute cartographie ne correspond pas à un réseau de sites ayant fonctionné de manière simultanée, avec des cas d'échecs, d'abandons, de déplacements de sites et, a contrario, de pérennités d'occupations ${ }^{59}$.

$\mathrm{Si}$ on se réfère à ce qui a été initié dans d'autres régions, chaque site devrait donner lieu à un relevé topographique qui permettrait de "déclasser » quelques mottes et de mieux appréhender certains sites et l'agencement de leurs défenses, voire de proposer une chronologie relative pour les retranchements les plus complexes $^{60}$. En Normandie, une approche micro-topographique a permis de reconstituer le tracé primitif de certains retranchements de terre occultés par des ouvrages de pierre postérieurs grâce à une démarche comparative entre fortifications de terre "fossilisées » sous bois et forteresses modernisées militairement et restées opérationnelles durant tout le Moyen Âge, comme le château de Gisors ${ }^{61}$. La topographie permet aussi de connaître les dimensions, les dispositions internes de certains bâtiments, les abords du château et la présence de basses cours. Cette démarche peut être menée conjointement avec l'examen du parcellaire et des microtoponymes observables sur le cadastre napoléonien, démarche que peut venir compléter l'archéologie aérienne ${ }^{62}$. Ces différentes approches sont parfois

${ }^{56}$ Renoux, Annie, « Châteaux, palais et habitats aristocratiques... », art. cité, p. 241.

${ }^{57} \mathrm{SALCH}$, Charles-Laurent, Dictionnaire des châteaux et des fortifications du Moyen Âge en France, Strasbourg, 1979.

${ }^{58}$ SÉRAPHIN, Gilles, Donjons et châteaux du Moyen Âge dans le Lot, Portet-sur-Garonne, 2014 ; BAUDRY, Marie-Pierre, Châteaux "romans " en Poitou-Charentes, XI ${ }^{\ominus}-X I^{\ominus}$ siècles, Cahiers du Patrimoine $n^{\circ}$ 95, La Crèche, 2011 ; http//cecab-chateaux-bourgogne.fr/.

${ }^{59}$ Les Boutteville, seigneurs du Faouët, disposaient ainsi de trois résidences vers les $X V^{\mathrm{e}}$ et $X V l^{e}$ siècles : le château du Faouët, la maison forte de Barrégan à deux kilomètres de distance et le "château » du Saint, bourg situé à 8 kilomètres au nord-ouest. Pour un exemple de réseau de châteaux et manoirs, voir JEANNERET, Lucie, " « Le réseau castral du Porhoët et du Rohan : mise en place et morphologie des sites fortifiés du $\mathrm{X}^{\mathrm{e}}$ au XIII ${ }^{\mathrm{e}}$ siècle ", Bulletin de la Société polymathique du Morbihan, t. CXL, 2014, p. 123-153.

${ }^{60}$ Comme pour le site de Domnaiche en Lusanger (Loire-Atlantique) par MEURET, JeanClaude, "Origines et débuts du manoir... ", art. cité, p. 87-90.

${ }^{61}$ Flambard-HÉRICHER, Anne-Marie, LePEUPLE, Bruno, « Topographie et prospection. Une approche renouvelée de l'étude des châteaux 1980-2006 ", Château Gaillard, t. XXIII, 2008, p. 189-204; LEPEUPLE, Bruno, "Du château au bourg castral en Vexin normand ( $\mathrm{X}^{\mathrm{e}}$ XII ${ }^{e}$ siècle) », dans FlAMBARD-HÉRICHER, Anne-Marie et LE MAHO, Jacques, (dir.), Château, ville et pouvoir au Moyen Âge, table ronde du CRAHM de 2008, Caen, 2012, p. 13-40.

${ }^{62}$ La prospection aérienne peut donner lieu à une fouille, voir MEURET, Jean-Claude, "Visseiche, vingt-cinq siècles d'aristocratie. De l'archéologie à l'écrit », Mémoires de la Société d'histoire et d'archéologie de Bretagne, t. LXXXIV, 2006, p. 131-180. 
combinées comme en Bourgogne, par le Centre de Castellologie de Bourgogne ${ }^{63}$. Dans le cas des manoirs bretons, l'équipe de Gwyn Meirion-Jones a déjà contribué à la datation de certains bâtiments grâce à l'utilisation conjointe de la dendrochronologie, de l'analyse architecturale et de l'étude approfondie des sources d'archives. Elle a ainsi pu retrouver des structures insoupçonnées dans les manoirs : tour, salle et bâtiments de charpente. Le recours à des bases de données, à la géomatique, au SIG (système d'information géographique) et au MNT (modèle numérique de terrain) a également permis à Hervé Mouillebouche de déterminer quels étaient les sites privilégiés d'implantation de l'habitat fortifié en Bourgogne ${ }^{64}$. Les seigneurs y ont initialement édifié leurs maisons fortes dans des sites présentant des prédispositions pour la défense et la surveillance des territoires mais aussi par la suite dans des zones déjà peuplées, en fonction des terres qu'ils détenaient et des expositions préconisées dans les traités d'agronomie antiques et médiévaux.

\section{L'exemple du Centre Ouest Bretagne : une pluralité de sites fortifiés}

Une prospection-inventaire a été menée sur plus 110 communes du CentreOuest Bretagne par l'archéologue Alain Provost, de 2002 à 2008, dans une zone jusque-là peu explorée, à cheval sur les départements du Finistère, du Morbihan et des Côtes-d'Armor ${ }^{65}$. Elle a permis de recenser près de 1500 sites archéologiques, dont près d'un tiers inédits, et de compléter les fichiers de la Carte archéologique nationale gérée par le Service régional de l'Archéologie (SRA) de Bretagne. Parmi ces ouvrages figurent, selon notre examen, environ 141 retranchements médiévaux : 15 châteaux de pierre, 26 mottes conservées, 17 mottes détruites, 21 maisons fortes, 6 tertres, 21 enceintes médiévales et 35 autres enceintes ou enclos présumés médiévaux. Ils sont les témoins d'un paysage castral varié dont l'état actuel reflète assez mal ce qu'il a pu être au Moyen Âge ${ }^{66}$.

\section{Carte des châteaux, maisons fortes, mottes et enceintes du Centre Ouest Bretagne}

II convient d'emblée de préciser que les manoirs n'ont pas été répertoriés par l'archéologue ni par le Service régional de l'Archéologie dans la mesure où ils le sont traditionnellement par les services de l'Inventaire régional de Bretagne. Les « entités

${ }^{63}$ Voir pour exemple, Mouillebouche, Hervé et Nouvellet, Yves, (dir.), Chastels et maisons fortes, actes des journées de castellologie de Bourgogne, t. v, 2013-2014, Chagny, 2015.

${ }^{64}$ Mouillebouche, Hervé et SAligny, Laure, «Topographie et habitats fortifiés en Bourgogne du Nord : implantation, orientation et surveillance », dans CAUCHIES, Jean-Marie et GUISSET, Jacqueline, (dir.), Le château autour et alentours XIVe-XVI siècles. Paysage, parc, jardin et domaine, actes du colloque d'Ecaussines-Lalaing (18-20 mai 2006), Turnhout, 2008, p. 29-55.

${ }^{65}$ Provost, Alain, Inventaire du patrimoine archéologique du Centre Ouest Bretagne, rapports des opérations de prospection-inventaire, 2002, 2003-2004, 2004-2005, 2005-2006, 2006-2007, 2007-2008, rapports dactylographiés destinés au Service régional de l'Archéologie de Bretagne et au Pays du Centre Ouest Bretagne.

${ }^{66}$ KeRnÉVEZ, Patrick, "Mottes castrales et châteaux forts... », dans Archéologie en Centre Bretagne, op. cit., p. 158-171. 
archéologiques » apparaissent sur la base en ligne " l'Atlas des Patrimoines » gérée par le SRA ${ }^{67}$ alors que les manoirs ressortissent à celle de "I'Inventaire du patrimoine culturel de Bretagne " tenue par les services de l'Inventaire ${ }^{68}$. À la suite des inventaires topographiques publiés pour les cantons de Carhaix-Plouguer, Le Faouët et Gourin en 1969 et 1975, une autre étude inédite a porté sur la " Haute Cornouaille » avec les cantons de Callac, Maël-Carhaix, Rostrenen et Saint-Nicolasdu-Pélem. Elle a permis de recenser, notamment grâce aux réformations des $X V^{e}$ et $\mathrm{XVI}^{\mathrm{e}}$ siècles, 140 manoirs dans 33 communes, soit une moyenne de 4,2 édifices par commune $^{69}$. Les densités observées témoignent de l'opposition entre l'Argoat et l'Armor et sont bien moindres qu'en Léon ou en Trégor car les territoires sont moins peuplés et moins riches. Le pourcentage de disparition est de $46 \%$ : on l'explique par un déclin démographique, une dissolution progressive des structures féodales et l'appauvrissement de la petite noblesse rurale à l'époque moderne qui a pu entraîner la ruine des manoirs et leur déclassement en fermes ${ }^{70}$.

Il a été impossible de cartographier tous les manoirs détruits : les zones vides de la carte correspondent à des sols peu fertiles ou à la présence d'espaces boisés. A contrario, les densités sont plus marquées le long des cours d'eau comme le Blavet et ses affluents avec des terrains humides, propices à l'établissement d'étangs, de viviers et de pêcheries. Par extrapolation, on peut avancer le chiffre de 400 manoirs pour l'ensemble du pays Centre-Ouest Bretagne; la moitié aurait totalement disparu. Une partie de ces résidences de la petite et moyenne aristocratie rurale pourraient ne pas être antérieure $a u X V^{e}$ et $a u X V I^{e}$ siècle. Au même titre que les églises et les chapelles, elles sont les témoins monumentaux de cet "âge d'or » de la Bretagne, les demeures de ces hobereaux ruraux, ces " sieurs ", qui sortent de la nuit documentaire bretonne à partir du milieu du $\mathrm{XIV}^{\mathrm{e}}$ siècle ${ }^{71}$. Certains sont promus à une vie meilleure par la grâce d'un bon mariage permettant de regrouper des terres, l'acquisition de fermes seigneuriales ou d'un office dans l'administration ducale. Ces fonctions pouvaient permettre, parfois au bout d'une ou deux générations, l'accès aux faveurs du prince et l'autorisation de fortifier, même modestement, sa maison. Toutes ces résidences ne sont pas nécessairement " manoir d'ancienneté », certaines ne sont que les témoins de l'ascension sociale d'anoblis, de bourgeois ou de paysans aisés aspirant à la noblesse dont les effectifs augmentent au $X \mathrm{VI} \mathrm{I}^{\mathrm{e}}$ siècle. Tous ces manoirs n'ont pas nécessairement succédé à des ouvrages plus anciens, mottes, enceintes ou maisons fortes ; dans certains cas, la demeure reconstruite s'est surimposée à un édifice antérieur dont ne subsistent que d'infimes traces.

L'étude des "ancêtres" des manoirs en Centre Bretagne se heurte à la carence des sources documentaires. Carhaix, ancienne capitale de la cité des

${ }^{67} \mathrm{http}$ ://atlas.patrimoines.culture.fr/atlas/trunk/.

${ }^{68} \mathrm{http}: / /$ patrimoine.bzh/.

${ }^{69}$ Les moyennes communales par canton s'élèvent à 10,5 pour Lesneven, 8,6 pour Ploudalmézeau, 11,2 pour les cantons de Pont-L'Abbé, Plogastel-Saint-Germain et Quimper et 12,3 pour l'ancienne châtellenie de Quimperlé, selon KERHERVÉ, Jean, « Temps des ducs, temps des rois. Manoirs et histoire », dans Le manoir en Bretagne..., op. cit., p. 28-43, note $\mathrm{n}^{\circ} 51$, p. 311.

70 DouARD, Christel, "Phénomènes de densité... ", art. cité, p. 44-53 (p. 46-47). II convient de corriger le chiffre donné de 110 manoirs en additionnant les chiffres annoncés pour chaque canton.

${ }^{71}$ Gallet, Jean, La Seigneurie bretonne (1450-1680), Paris, 1983, p. 79-81 et 113-118. 
Osismes, reste indiscutablement un important lieu de pouvoir, celui des comtes de Poher, à l'origine de la lignée royale de Bretagne au I $X^{\mathrm{e}}$ siècle. Tenue ensuite par les comtes de Cornouaille, la ville est aux mains des ducs de Bretagne au début du $\mathrm{XIII}^{\mathrm{e}}$ siècle. Les généalogistes ont fait de plusieurs lignages nobles un « ramage des comtes de Poher », comme pour les Kergorlay, les Rostrenen, les Plusquellec et les seigneurs de Mur-de-Bretagne, ce qui reste invérifiable ${ }^{72}$. Nous sommes à peine mieux renseignés sur Périou, fils cadet du comte de Cornouaille, qui donna son nom au château de La Roche-Périou implanté à Priziac vers le milieu du $\mathrm{Xl}^{\mathrm{e}}$ siècle tandis que son successeur, Guigon, aurait fondé celui de Guémené, siège du KémenetGuégant, une génération plus tard. Seules les donations du vicomte Tanguy qui fonde un prieuré près de son château de La Roche à Cléden-Poher, ou celle de Simon, fils de Cariou, mortellement blessé lors du siège de cette forteresse, au début $\mathrm{du} \mathrm{XII}$ e siècle, permettent de rattacher cette puissante motte au lignage vicomtal de Poher, disparu au début du XIII ${ }^{e}$ siècle, et à un épisode guerrier ${ }^{73}$. Les actes des ducs de Bretagne sont encore peu nombreux lors de la période féodale et les lignages qui émergent à cette époque, comme les Kergorlay, les Rostrenen ou les Plusquellec, n'ont pas conservé dans leurs archives de documents antérieurs au XIII siècle. Le livre des Ostz de 1294 ne nous livre qu'un aspect tardif et incomplet de la hiérarchie féodale: les seigneurs de Kergorlay et de Rostrenen fournissent chacun deux chevaliers, celui de La Roche-Helgomarch doit servir durant 15 jours seulement ${ }^{74}$. Certains châteaux n'apparaissent dans l'histoire qu'au moment de la guerre de Succession de Bretagne dans les chroniques de Jean Froissart: ils sont utilisés comme "forts " et assiégés, comme ceux de La Roche-Périou, Le Faouët et Guéméné-sur-Scorff, en $1342^{75}$.

Seul l'examen du réseau castral vers le $\mathrm{XIII}^{\mathrm{e}}$ siècle nous renseigne sur l'émergence et la réussite relative de certains lignages. II est dominé par les sept forteresses qui ont donné naissance à une agglomération : Carhaix et Châteauneuf sont aux mains des ducs, Corlay et Guémené-sur-Scorff sont passées dans celles des Rohan, Rostrenen, Callac et Le Faouët ont appartenu à des lignages de la moyenne aristocratie dont c'était la principale forteresse ${ }^{76}$. Pour les autres, nous demeurons tributaires de l'examen morphologique des structures subsistantes, de la présence avérée de bâtiments de pierre et des sites d'implantation pour appréhender le rôle qu'ils ont pu jouer. Quelques fouilles récentes ont toutefois permis de lever le voile sur quatre enceintes d'époque carolingienne : Bressilien à Paule, Goarem-arManec'h à La Feuillée, Kergoac'h-Villérit à la lisière de Mellionnec et de Ploërdut et Talhoët à Langoelan ${ }^{77}$. Ces ouvrages associent des bâtiments aristocratiques

${ }^{72}$ Potier de Courcy, Pol, Nobiliaire et armorial de Bretagne, 3 vol., Nantes-Paris, 1862, rééd., 2 vol., Mayenne, 1993, t. II, p. 89, 315, 400 et 512.

${ }^{73}$ DE Courson, Aurélien, Cartulaire de l'abbaye bénédictine de Redon en Bretagne, Paris, 1863, n CCCLXXII, p. 332-333 ; MAÎTRE, Léon et De BeRTHOU, Paul, Cartulaire de l'abbaye de Sainte-Croix de Quimperlé, $2^{e}$ éd., Rennes-Paris, 1904, $n^{\circ}$ LXXI, p. 216-217.

${ }^{74}$ MoRVAN, Frédéric, "Le Livre des Ostz (1294). Un éclairage sur les rapports du duc avec la noblesse bretonne à la fin du XIII e siècle ", dans KERHERVÉ, Jean, (dir.), Noblesses de Bretagne du Moyen Âge à nos jours, Rennes, 1999, p. 37-88 (p. 83-85).

${ }^{75}$ Les chroniques de Sire Jean Froissart, édition J.A.-C. Buchon, 3 vol., Paris, 1835, t. I, p. $151-158$.

${ }^{76}$ KeRNÉVEZ, Patrick, « Mottes castrales et châteaux forts... », art. cité, p. 159-163.

${ }^{77}$ LE GALL, Joseph et LeRoY, Benjamin, « Le haut Moyen Âge... », art. cité, p. 129-133. Les rapports de fouilles sont consultables en ligne sur le site de la bibliothèque numérique du 
résidentiels et des dépendances, parfois au sein d'ouvrages bipartites. Le premier a précédé un logis du $\mathrm{XIV}^{\mathrm{e}}$ siècle distant de quelques dizaines de mètres auquel a succédé un manoir implanté à 150 mètres au $X V V^{e}$ siècle, ce qui autorise à penser que ces « enceintes constituent peut-être les sièges des premières seigneuries bretonnes $^{78}$ ". Les fouilles de l'important site de Saint-Symphorien à Paule ont mis en évidence un autre exemple de postérité manoriale entre la petite " motte " à bassecour de Saint-Eloy occupée entre le $\mathrm{XI}^{\mathrm{e}}$ et le XIII siècle et le manoir du même nom distant de 200 mètres $^{79}$. Dans les deux cas, les enceintes avaient été totalement nivelées, ce qui laisse augurer d'autres découvertes par l'archéologie aérienne, parfois vitale pour bien appréhender la globalité des résidences aristocratiques médiévales. La filiation est parfois bien plus évidente quand une petite enceinte circulaire ou une motte est voisine d'un manoir et d'un moulin portant un même nom que l'on retrouve dans les registres de réformation des $\mathrm{XV}^{\mathrm{e}}$ et $\mathrm{XVI} \mathrm{I}^{\mathrm{e}}$ siècles (Kermain à Langonnet, Quénécadec à Lennon).

D'un point de vue topographique, deux caractéristiques apparaissent: la recherche du perchement ou celle de la protection des cours d'eau. Quelques sites sont de véritables nids d'aigle érigés à l'extrémité d'un affleurement rocheux, comme Castel-Cran à Plélauff ou Roc'h-Castel à Saint-Goazec dont la perception dans le paysage devait être forte et l'accès plus problématique. On a aussi privilégié les implantations sur des éperons, parfois retaillés pour les séparer du relief voisin (La Roche Piriou, Priziac), isolés par un rempart de barrage ce qui prête parfois à confusion avec des ouvrages de l'Âge du Fer ou encore refaçonnés pour réaliser une motte d'extrémité d'éperon (La Roche, Cléden-Poher ; Le Valy, Locmaria-Berrien). II s'agit parfois d'un promontoire fort modeste qui sert d'assiette à un petit château de pierre, une maison forte, de 1000 à 1500 mètres carrés seulement (Castel-Pennod, Lothey ; Kermargon, Lennon). Dans d'autres cas, on a utilisé un pointement rocheux au-dessus d'un petit vallon pour y adosser une forteresse (La Roche-Helgomarc'h, Saint-Thois) ou le coiffer par un ouvrage, enceinte (Milin-ar-Hastel, Saint-GillesPligeaux) ou motte (ar Hastel, Peumerit-Quintin). D'autres somment des collines d'où l'on peut bénéficier d'un large panorama (Saint-Gildas, Carnoët ; La Roche-Droniou, Calanhel). Le caractère contraignant de quelques emplacements suffit à comprendre certains déplacements intervenus postérieurement. La protection de l'eau a parfois prévalu et elle entoure certains retranchements sur la totalité de leur pourtour comme pour le château de Pestivien à Bulat-Pestivien. Des petites enceintes ou des terrasses sont aujourd'hui encore isolées dans des prairies à quelque distance du hameau le plus proche (Le Loc'h, Peumerit-Quintin; Guernodic, Le Croisty). Des travaux d'aménagement ont parfois permis de réaliser des étangs qui ont pu contribuer à la défense d'une place (Castel Bel-Air, Priziac ; Pontcallec, Berné ; Corlay ; Rostrenen).

L'ampleur de certains retranchements et la présence de pierre suffisent parfois à comprendre qu'un site castral a pu jouer un rôle notoire quoique méconnu. II en est ainsi de certains sites de mottes comme La Roche à Cléden-Poher, le château du vicomte Tanguy au début du $\mathrm{XII}^{\mathrm{e}}$ siècle mais aussi les retranchements de

Service régional d'archéologie de Bretagne, bibliotheque.numerique.sra-bretagne.fr.

${ }^{78}$ ID., ibid., p. 131 ; sur l'antériorité des enceintes par rapport aux mottes, voir DECAËNS, Joseph, « Les enceintes circulaires... », art. cité, p. 47-51.

${ }^{79}$ LE GALL, Joseph, "Une résidence aristocratique des VIII $-1 X^{\mathrm{e}}$ siècles au cœur de la Bretagne. L'enceinte de Bressilien à Paule (Côtes-d'Armor) », dans Les élites et leurs résidences en Bretagne..., op. cit., p. 125-138 (p. 136-137). 
Castel-ar-Valy à Locmaria-Berrien ou de Rospellem à Carnoët. L'absence d'un manoir proche peut laisser croire à une destruction précoce ou à une extinction du lignage. Cela est attesté pour la motte de Kergorlay à Motreff et le château de Roc'hCastel à Saint-Goazec, chefs-lieux de seigneuries passées dans les mains du lignage de Laval: ces deux forteresses sont en ruine dès le milieu du $\mathrm{XV}^{\mathrm{e}}$ siècle ${ }^{80}$. D'autres ont pour postérité un manoir édifié à proximité et confié, en l'absence de leur propriétaire détenteur de plusieurs fiefs, à la garde d'un intendant chargé de prélever les rentes seigneuriales, comme à La Roche-Helgomarc'h à Saint-Thois au $\mathrm{XVI}^{\mathrm{e}}$ siècle ${ }^{81}$. Des manoirs sont érigés à courte distance comme celui de Kermain à Langonnet ou parfois même au sein de sa basse-cour, comme à Cravial en Lignol, bel exemple de ces " manoirs à motte » découverts par dizaines dans le comté de Rennes $^{82}$. Dans certains cas, une construction moderne a pu effacer les dispositions d'origine, comme pour les châteaux urbains, tel Guéméné-sur-Scorff reconstruit aux $X V^{e}$ et $X V I^{e}$ siècles: les logis ont alors été aménagés dans l'ancienne basse-cour d'une présumée motte ${ }^{83}$.

On recense également une pléthore d'ouvrages de dimensions moindres, parfois classés dans la catégorie "fourre-tout» des maisons fortes. Le retranchement de Barrégan au Faouët, désigné comme "manoir » en 1426, qui correspond à un logis de pierre cantonné de deux tours précédé d'un ouvrage circulaire identifié à une " motte » et de retranchements de terre et de pierre, y côtoie des terrasses où l'on observe plus que des pierres éparses comme Le Loc'h à Peumerit-Quintin, le petit château de Bel-Air à Priziac ou encore le Castel-Grannec à Landeleau qui présente les caractéristiques d'un château fortifié du $\mathrm{XVI} \mathrm{l}^{\mathrm{e}}$ siècle bordé de profondes douves. II reste parfois délicat également d'identifier de grosses enceintes de terre désignées par la tradition comme étant un "château " à des ouvrages signalés comme "manoir » dans les réformations du XV siècle comme Kerjean à Paule ou Le Pélinec à Canihuel. Dans certains cas, on peut même présumer que le "manoir » mentionné dans la réformation de 1426 est encore localisé sur la motte du même nom comme à Kergorlay en Motreff et au Moulin de Mezle à Maël-Carhaix ${ }^{84}$. II en est de même pour ceux de Pestivien à Bulat-Pestivien ou de La Roche-Droniou à Calanhel où le "manoir " correspond à ce que nous avons recensé comme "château ». Le vocabulaire utilisé à l'époque est resté fluctuant d'un acte à l'autre ${ }^{85}$.

${ }^{80}$ MOUSSET, Albert, Documents pour servir à l'histoire de la maison de Kergorlay en Bretagne, Paris, 1921, p. 124-125.

${ }^{81}$ Du HALGouËT, Hervé, Inventaire des archives du château du Grégo (1343-1830), SaintBrieuc, 1913, p. 160.

${ }^{82}$ BRAND'HONNeUR, Michel, Manoirs et châteaux dans le comté de Rennes..., op. cit. .

${ }^{83}$ KERNÉVEZ, Patrick, "Les trois vies du château de Guémené-sur-Scorff ", Actes du colloque de Guémené-sur-Scorff du 29-30 avril 2011, Gourin, 2013, p. 118-134 (p. 128).

${ }^{84}$ TORCHET, Hervé et TORCHET, Yann, Réformation des fouages de 1426. Diocèse ou évêché de Cornouaille, op. cit., p. 49, 156-159.

${ }^{85}$ JONES, Michael, "The naming of parts : remarques sur le vocabulaire des résidences princières et seigneuriales en Bretagne au Moyen Âge », dans RENOUX, Annie, (dir.), "Aux marches du palais" ". Qu'est-ce qu'un palais médiéval? Données historiques et archéologiques, actes du congrès du Mans et de Mayenne (9-11 septembre 1999), Le Mans, 2001, p. 45-54. 
Les retranchements du Centre Ouest Bretagne n'échappent pas à la diversité qui caractérise les ouvrages fortifiés de terre, de bois et de pierre du Moyen Âge. Les châteaux de pierre les plus importants ont souvent été détruits, par vétusté ou du fait de l'urbanisation et de leur exploitation comme carrière de pierres, comme pour les châteaux de Callac et de Guémené au début du $X X^{e}$ siècle. Peu d'ouvrages ont fait l'objet d'explorations archéologiques ${ }^{86}$ : on peut se réjouir des investigations récentes menées sur quelques enceintes du haut Moyen Âge et appeler de nos vœux qu'elles s'étendent, dans un avenir proche, à l'étude de quelques-unes des mottes du Poher, comme La Roche à Cléden-Poher ou Kergorlay à Motreff. II conviendra également d'explorer enfin le «Tour du Chastel » de Carhaix afin de déterminer de quand datent les défenses de cette ville et quelle était la nature précise de son château. Les autres, mottes, enceintes, maisons fortes et manoirs attendent encore un inventaire exhaustif et des notices, alliant relevé topographique, lecture du bâti et étude des sources historiques. L'étude de leurs vestiges devra être étendue à leur environnement: marques de châteaux, moulins, garennes, bois, fourches patibulaires, métairies, parcellaires... comme cela a pu être réalisé en d'autres lieu ${ }^{87}$. Ces réexamens devront notamment être entrepris dans le cadre des valorisations préconisées pour une quinzaine de sites de ce territoire ${ }^{88}$.

${ }^{86}$ Citons pour la Bretagne les fouilles programmées menées au Guildo (Créhen, Côtesd'Armor) par Laurent Beuchet, à La Roche-Maurice (29) par Jocelyn Martineau puis Ronan Perennec, à Suscinio (Sarzeau, Morbihan) par Karine Vincent, outre quelques fouilles préventive d'ampleur, comme celles de Laurent Beuchet au château de Guingamp (Côtesd'Armor) ou sur le site de Chasné-sur-Illet (Ille-et-Vilaine) qui a révélé la succession d'un habitat du haut Moyen Âge, d'une motte et d'un " manoir ». Les rapports de fouilles sont consultables en ligne sur le site de la bibliothèque numérique du Service régional de l'Archéologie de Bretagne, bibliotheque.numerique.sra-bretagne.fr.

${ }^{87}$ CAUCHIES, Jean-Marie et GUISSET, Jacqueline, (dir.), Le château autour et alentours $X I V^{e}-X V I^{e}$ siècles..., op. cit. ; ID., Lieu de pouvoir, lieu de gestion. Le château auX $X I I^{e}-X V{ }^{e}$ siècles: maîtres, terres et sujets, actes du colloque d'Ecaussines-Lalaing (mai 2009), Turnhout, 2011.

${ }^{88}$ LANCTUIT, Bernard et HeRBAULT, Claudie, Archéologie et paysages, un projet culturel pour un territoire. Étude de programmation et planification de travaux de mise en valeur de sites archéologiques, rapport dactylographié destiné au Pays du Centre Ouest Bretagne, novembre 2013, p. 8, 29 et 54-55. Les sites retenus sont les châteaux de Châteauneuf-du Faou, Corlay et Guémené-sur-Scorff ; la maison forte de Barrégan au Faouët ; les enceintes médiévales de Lesneven à Châteauneuf-du-Faou, Goarem-ar-Manech à La Feuillée et Castel-Vouden à Roudouallec ; les mottes de La Roche à Cléden-Poher, Rospellem et SaintGildas à Carnoët, Castellaouenan à Paule, Camp d'Artus au Huelgoat, Kergorlay à Mottreff et Kermain à Langonnet. 